\title{
«Der Medicus» im Kino: ein medizinisches Märchen
}

Eberhard Wolff

eberhard.wolff[at]saez.ch
Die älteren Semester unter uns erinnern sich sicherlich: In den 80er Jahren schied der 850-SeitenRoman die Geister. Die einen verschlangen ihn möglichst an einem Stück, die anderen blieben auf Distanz zum Millionenseller «Der Medicus» von Noah Gordon. Erst jetzt ist das Buch verfilmt worden und zu Weihnachten in die Kinos gekommen. Das Publikum hat ihn nicht schlecht aufgenommen, die Kritiker sind dagegen eher skeptisch.

Es war ein immenses cineastisches Unterfangen. Herausgekommen ist ein bildgewaltiger Abenteuerfilm in Überlänge. Grosses Cinemascope-Kino mit atemberaubenden Landschaften und überzeugenden Schauspielern bis hin zum Gandhi-Darsteller Ben Kingsley als mittelalterlichem arabischem A-PromiArzt Ibn Sina alias Avicenna. Auch wenn Kritiker den Film zu glatt finden: Einen Kino-Besuch kann ich durchaus empfehlen oder in ein paar Monaten die Anschaffung einer Blu-ray-Version, aber nur, wenn Sie mindestens einen 42-Zoll-Bildschirm zu Hause haben.

Die seriöse SÄZ ist kein Kino-Magazin. Deshalb werfen wir hier einen speziellen Blick auf den medizinischen Plot, der sich wie eine Perlenkette durch den Film zieht. Die Geschichte erzählt von Rob Cole, der als kleiner Bub im England des 11. Jahrhunderts seine Mutter durch die «Seitenkrankheit» verliert. Rob lernt bei einem Bader das Handwerk des Purgierens und Amputierens, aber er will mehr wissen. Er kämpft, bis sein inzwischen erblindeter Lehrmeister durch einen jüdischen Arzt mittels Starstich geheilt wird. Dann macht er sich auf die lebensgefährliche Reise ins ferne persische Isfahan zum berühmtesten Arzt seiner Zeit Ibn Sina, gibt sich als Jude «Jesse» aus und findet Rebecca, die Frau seines Lebens.

In Isfahan wird Jesse bei Ibn Sina zum Arzt, kühlt bei der Pestepidemie das Fieber mit Eis und Wadenwickeln. Er entdeckt Floh und Ratten als Krankheitsüberträger und kann die Epidemie stoppen. Auf der Suche nach den Ursachen der «Seitenkrankheit» obduziert er einen Verstorbenen, fertigt anatomische Zeichnungen an und entdeckt den entzündeten Blinddarm. Als Showdown operiert er in seinem Meisterstück dem Schah selbst relativ erfolgreich den Blinddarm. Angesichts seiner drohenden Hinrichtung bekennt er sich zum Christentum und rettet die Juden Isfahans vor dem Terror der Mullahs und Seldschuken. Am Ende führt Rob mit Rebecca ein Hospital in London.

Und bereits an dieser Stelle komme ich zum eigenen Showdown meiner Filmbesprechung: Bitte, bitte, fangen wir gar nicht damit an, gegeneinander aufzurechnen, was an dem Film medizinhistorisch verbürgt ist und was nicht! Die Macher haben sich um viel historische Authentizität im Detail bemüht, aber wie eine zweite Perlenkette ziehen sich auch schreiende Anachronismen durch den Film, die in die nachfolgenden acht Jahrhunderte gehören, von der Leichensektion über den Pesterreger und die Entdeckung des Blutkreislaufs bis zur Blinddarmoperation.

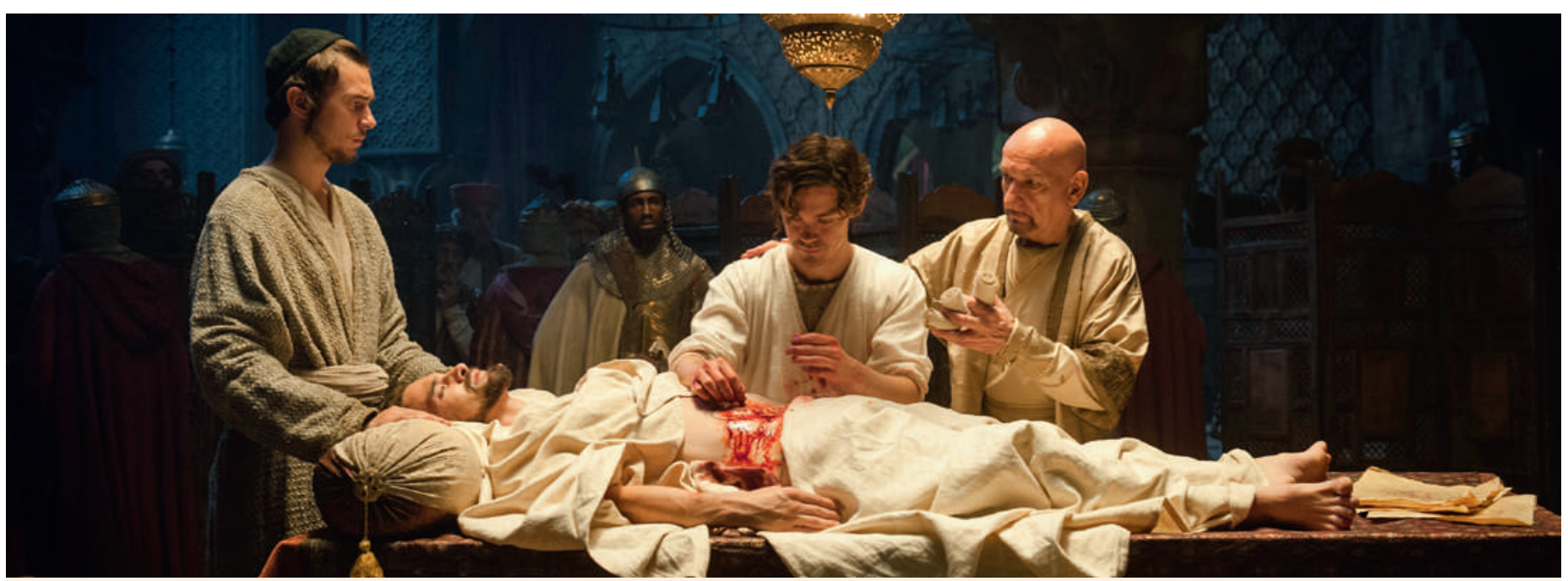

Tom Payne als «Medicus» (Mitte) und Ben Kingsley (rechts) bei der Operation eines Blinddarms. Auch wenn es nicht so aussieht: Alles geht gut - erst einmal. 


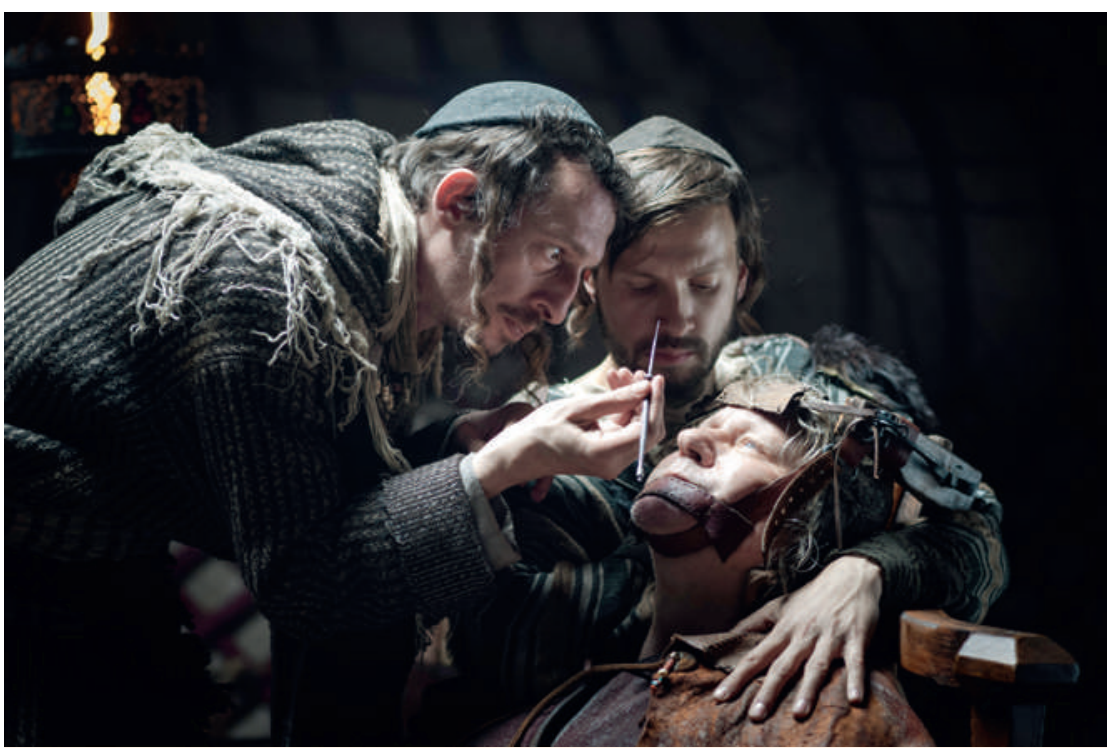

Der Bader (Stellan Skarsgård) lässt sich von einem jüdischen Arzt den Star stechen. Auch hier geht alles gut, sogar auf Dauer. telalterliche Sterbestatistik und Letalitätskurve mit Kreide an der Wand) von falschem Aberglauben. Dabei sind sie natürlich auch ganzheitlich, wenn Ibn Sina den «ganzen» Patienten behandeln will und Jesse als guter Assistenzarzt einen Todgeweihten am Krankenbett bis zum Ende begleitet. Schliesslich gehört eine Prise übersinnlicher Seher-Qualitäten zum absoluten Arzt - wie bei Jesse alias Rob, der den Tod sicher voraussagen kann. Und als Topping klassischer Medizin-Utopien muss der Arzt natürlich auch ein wenig Superhero sein. Das hat alles viel mit Märchen und wenig mit Geschichte zu tun.

Der Film erzählt darüber hinaus das grosse historische Märchen, dass unsere heutige Medizin das Ziel und die Erfüllung aller Geschichte ist. Sinngemäss sagt Ibn Sina in einer Szene: «Heute wissen wir das noch nicht, aber in hundert oder tausend Jahren vielleicht.» In diesem Märchen ist das Früher nur entweder die Vorahnung des Heute, wie bei den Heldenfiguren, oder das Gegenteil des Heute, wie das düstere, dreckige und dumme Mittelalter, das im Film bis über die $\mathrm{Sch}(\mathrm{m})$ erzgrenze hinaus in Szene gesetzt ist.

«Der Medicus» ist eine heutige Utopie einer guten Medizin ohne TARMED, Guidelines, DRG und CME-Punkte, Ethik-Kommissionen und, Gott bewahre, Teilzeitärzt(inn)en! Und wenn das noch so weltfremd ist: Können wir das Kino dafür schelten, dass es eine Traumfabrik ist?

Der französische Soziologe Maurice Halbwachs, umgekommen $1945 \mathrm{im} \mathrm{KZ} \mathrm{Buchenwald,} \mathrm{hat} \mathrm{sinnge-}$ mäss einmal gesagt, dass das, woran wir uns erinnern, mehr mit unseren heutigen Ideen als mit dem Gewesenen $\mathrm{zu}$ tun hat. Auch die nichtmedizinischen Utopien im historisierenden Medicus-Film zeigen das: Heutige Wunschvorstellungen und heutige Horrorszenarien dampfen gleichsam aus dem Film: die friedliche Multikulturalität und das Nebeneinander zumindest von Juden und Mohammedanern im Nahen Osten oder die sich selbst befreiende Rebecca-Frau. Auch die aufkeimende Mullah-Herrschaft im mittelalterlichen Isfahan scheint direkt von den Bildern heutiger Nachrichtensendungen abgemalt zu sein.

Ich empfehle Weisskitteln und verwandten Berufen, den Film wie folgt zu verwenden: zurücklehnen, geniessen, träumen - und nach dem Kino in diese Welt zurückkehren, die TARMED, Guidelines, DRG und CME-Punkte, Ethik-Kommissionen und Teilzeitärzt(inn)en nicht ohne Grund hervorgebracht hat. Dabei wünsche ich viel Unterhaltung.

«Der Medicus». Nach dem Roman von Noah Gordon. Deutschland 2013. 150 Minuten. Regie: Philipp Stölzl. Mit Tom Payne, Stellan Skarsgård, Olivier Martinez, Emma Rigby und Ben Kingsley. 